\title{
Los servicios digitales: un valor añadido en la gestión de la calidad en bibliotecas y centros de documentación en ciencias de la salud
}

\author{
Ana Nieves Millán Reyes \\ María Juana Millán Reyes \\ Universidad de Granada (España)
}

\section{Resumen}

Se realiza un estado de la cuestión sobre la situación actual de los servicios digitales de las bibliotecas y centros de documentación públicos en ciencias de la salud de ámbito estatal. La muestra recoge aquellos centros hospitalarios públicos que cuentan con página web para el servicio de biblioteca y centro de documentación en España. Se analiza la presencia o ausencia de servicios digitales, sus tipos, cuáles son los más frecuentes, etcétera. Se distinguen como más frecuentes los de información.

Palabras clave: Calidad. Centros de documentación. Bibliotecas. Ciencias de la salud. Servicios digitales. TQM. EFQM.

\section{Abstract}

A review article on library and information centers quality is presented, analysing the digital services on Internet of health sciences libraries and information centers in Spain. The sample studied includes public hospitals that have a website for the library an information centre service in Spain. The presence or absence of digital services, type and number of services and others are also analysed. Information services are the most frequent ones.

Keywords: Quality. Libraries. Information centres. Digital services. Health sciences. TQM. EFQM.

\section{Introducción}

La gestión de la calidad se une a un cambio de mentalidad en la sociedad, donde la atención se centra en la satisfacción del usuario/cliente. Se convierte en una estrategia organizativa y un método de gestión que involucra al personal para mejorar continuamente la eficacia de la institución (en nuestro caso, las bibliotecas y centros de documentación de ciencias de la salud) u organización para satisfacer al cliente. 
En esta línea, el sistema tratará de mejorar los productos y servicios que ofrece a la comunidad a la que sirve, de manera que se exige la satisfacción del usuario no solo con la información suministrada, sino también con la presentación y la prestación del servicio asociado a tal suministro (Lancaster y Pinto, 2001).

De este modo, en la época actual, marcada por los planteamientos derivados de la filosofía de la calidad, la gestión, la sociedad de la información, y caracterizada por las tecnologías, el cambio y la agilidad, el hecho de tener que adaptarse a los nuevos retos, innovar, ha llevado a multitud de instituciones, incluidas las bibliotecas y los centros de documentación, a prestar sus servicios a través de estos medios y herramientas. Los actuales profesionales de las ciencias de la salud tienen unas necesidades informativas diferentes.

Esta serie de cambios ha generado la demanda de nuevos planteamientos en relación con nuestras instituciones: existencia, pertinencia, formas de persistencia, rol profesional, nuevas funciones, importancia cobrada en nuestros días por la generación y adquisición de conocimiento, etcétera.

Los servicios de información no pueden quedarse al margen de esta revolución; aunque sigan manteniendo su vertiente física, han de generarse a través de la tecnología nuevos servicios digitales, virtuales, que vendrán a sumarse a las tareas tradicionales de organización, difusión, tratamiento, etcétera, facilitando el acceso a la información y una mayor satisfacción del usuario, y generando así un valor añadido.

Las ventajas son enormes, puesto que el empleo de redes, tanto universales (Internet) como internas (intranets), nos permite ofrecer servicios fuera del entorno habitual, con las ventajas que ello conlleva: rapidez, comodidad para el usuario, descongestión de las bibliotecas, rentabilización de recursos y superación de barreras. Sin olvidar las limitaciones con que nos encontramos: problemas legales de acceso al documento, necesidades de personal especializado, adquisición de software y hardware, etcétera.

\section{Objetivos}

El objetivo de este trabajo es analizar la presencia de bibliotecas y centros de documentación en ciencias de la salud en España hasta el momento en que nos encontramos, con la introducción de las nuevas tecnologías de información y comunicación.

De este modo se pretende analizar las posibles transformaciones a la hora de prestar servicios de calidad a través de estos medios (Internet y las páginas webs) como un valor añadido no solo para las bibliotecas, sino también para la institución sanitaria a la que se encuentran vinculadas, estudiar los servicios digitales que ofrecen dichas bibliotecas a través de sus páginas webs y ver cuáles son los más

Scire. $13: 2$ (jul.-dic. 2007) 59-65. ISSN 1135-3716. 
ofrecidos, así como determinar los centros que cumplen las condiciones de selección, es decir, que pertenecen a la Seguridad Social o dependen de la Comunidad Autónoma y además tienen página propia para analizar los servicios digitales que prestan (información general, etcétera), propuestos siguiendo una tipología expuesta a continuación.

\section{Metodología}

Para llevar a cabo el estudio se estableció como criterio de selección la búsqueda en el Catálogo Nacional de Hospitales del Ministerio de Sanidad, por comunidades autónomas, de aquellos centros que perteneciesen a la Seguridad Social o dependiesen de cualquiera de las comunidades autónomas españolas. De este marco maestral se obtuvo una población de 236 centros que respondían a alguno de estos criterios.

Posteriormente se hizo un rastreo o browsing en Internet para determinar qué centros tenían página web. Una vez identificados estos, se buscó la página web de la biblioteca o centro de documentación clínica con el fin de registrar los servicios digitales correspondientes. Para la localización de las diferentes páginas se emplearon desde buscadores (Altavista o Google) hasta diferentes directorios como los que se pueden ver en la tabla I.

\begin{tabular}{|l|l|}
\hline Nombre del recurso & URL \\
\hline $\begin{array}{l}\text { Hospitales de España. } \\
\text { Directorio UCM }\end{array}$ & http://www.ucm.es/BUCM/med/0530.htm \\
\hline Bibliotecas biomédicas & $\begin{array}{l}\text { http://www.cs.urjc.es/biblioteca/recursos/medicina/ } \\
\text { Bibliotecas\%20biomedicas/bibliotecas.htm }\end{array}$ \\
\hline $\begin{array}{l}\text { Cátalogo Nacional de Hospitales. } \\
\text { Ministerio de Sanidad y Consumo. }\end{array}$ & http://www.msc.es/Diseno/sns/sns_sistemas_informacion.htm \\
\hline Hospitales. Comunidad de Madrid & http://www.madrid.org/sanidad/enlaces/hospitales.htm \\
\hline
\end{tabular}

Tabla I. Directorios empleados para el rastreo de las páginas webs tanto de las instituciones hospitalarias como de sus bibliotecas. (Fuente: elaboración propia)

Algunos de estos servicios digitales objeto de análisis fueron los siguientes (los relacionados con las adquisiciones no se estudiaron, pues normalmente se trata de servicios articulados a través de la intranet): $a$ ) información general: horarios, directorio, reservas de sala de trabajo; $b$ ) normativa, FAQ, guías, noticias, sugerencias; c) préstamo interbibliotecario, reservas, renovaciones de préstamos, consulta de préstamos; $d$ ) acceso: alerta informativa, formación de usuarios, DSI, catálogos, bases de datos, revistas electrónicas, sumarios electrónicos, información bibliográfica; e) otros servicios: exposiciones virtuales, recursos temáticos, bibliografía Scire. $13: 2$ (jul.-dic. 2007) 59-65. ISSN 1135-3716. 
recomendada (en este apartado se incluyó un criterio más: el de "error de conexión" / "en construcción" para aquellas bibliotecas que dieron error a la hora de acceder y las que se encontraban en fase de construcción en relación con el resto de servicios digitales a incluir).

\section{Resultados}

Ante todo, se puede decir que resultó un poco tedioso que el porcentaje de hospitales públicos dependientes de la Seguridad Social o bien de alguna comunidad autónoma que en 2005 contaban con página web en Internet fuera todavía de un $26 \%$; de ellos, solo un $17 \%$ tenían página web de la biblioteca o centro de documentación para prestar alguno de los servicios a través de medios digitales, electrónicos, etcétera. El mayor porcentaje (57\%) venía asociado a la carencia de página web hospitalaria como medio de difusión y valor añadido, como se puede ver en la figura 1.

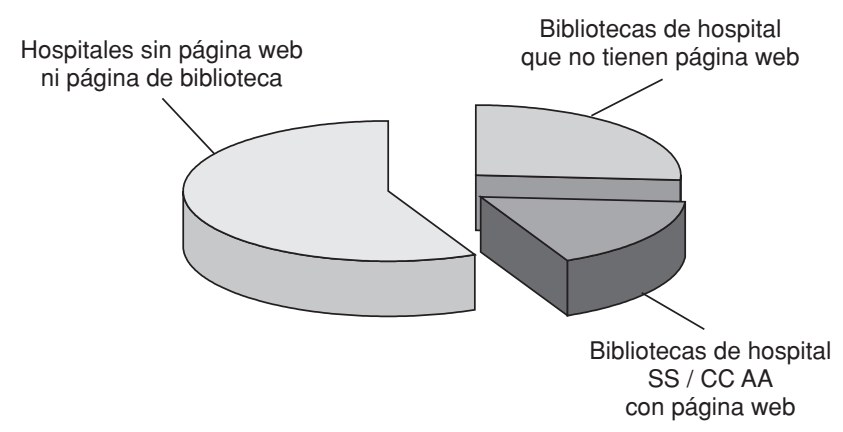

Figura 1. Porcentaje de hospitales y bibliotecas o centros de documentación que tienen página web con respecto al resto. (Fuente: elaboración propia)

Con respecto a los servicios prestados, podemos ver que los más destacados son, en un primer momento, los servicios de acceso (destaca el acceso al catálogo, las bases de datos y las revistas electrónicas) y con los de información general (horario, directorio de personal, etcétera), seguidos de los servicios de préstamo (lo menos frecuente en este caso son las reservas de préstamos por Internet y las consultas sobre estos), y por último de otros servicios (entre los que destacan los recursos temáticos y, en menor medida, las exposiciones virtuales y bibliografías recomendadas). Véase la figura 2.

Scire. 13 : 2 (jul.-dic. 2007) 59-65. ISSN 1135-3716. 


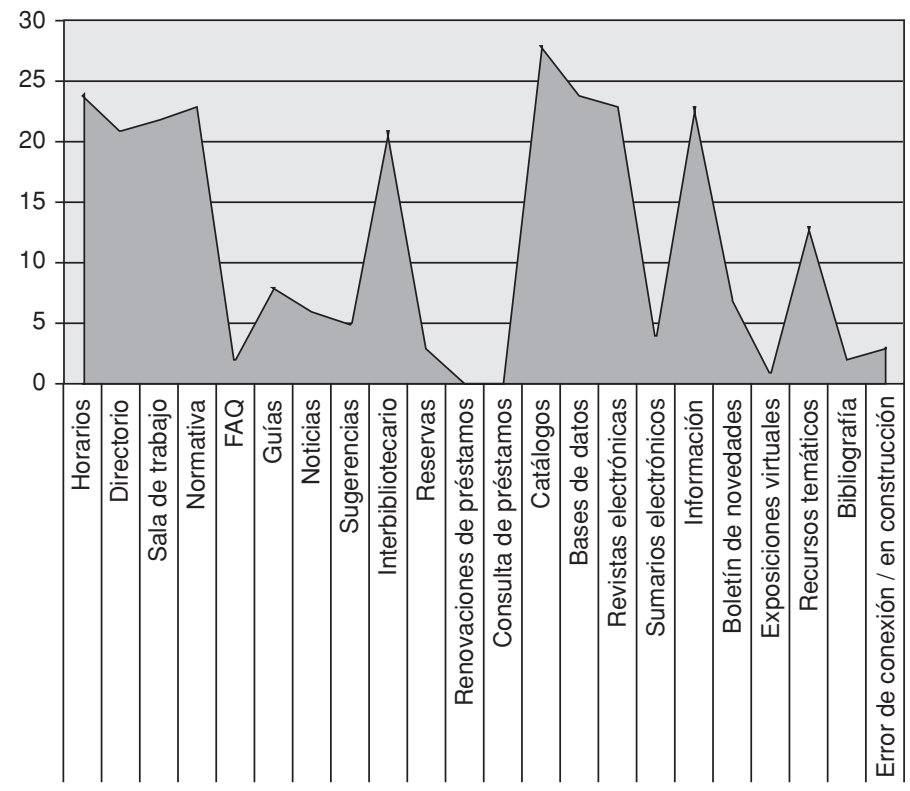

Figura 2. Comparativa entre los distintos servicios digitales ofrecidos por las bibliotecas o centros de documentación de hospitales con página web.

(Fuente: elaboración propia)

\section{Conclusiones}

En primer lugar, hay que destacar que con la aparición de las nuevas tecnologías parece haberse incrementado la presencia de las instituciones hospitalarias en la Red de Redes (Internet) como resultado de un nuevo sistema de gestión en estas instituciones, impregnadas de las nuevas filosofías de gestión y de la calidad. Se intenta pasar, como nos dice María Pinto (1994), "hacia una gestión basada en los procesos, productos y resultados a través del denominado ciclo de calidad, proponiendo planes de mejora y optimización de recursos". Nos consta que en estudios anteriores realizados en 2003, dos años antes que el nuestro, el número de bibliotecas empleado en el estudio era menor, lo que nos indica un pequeño crecimiento, como hemos dicho anteriormente, aunque hemos de tener en cuenta que los criterios de selección eran diferentes, y la finalidad también.

Sin embargo, muchas de estas instituciones, aun en la línea de estos planteamientos, olvidan que las nuevas tecnologías son un medio de difusión, y hallamos todavía un pequeño número de centros que no nos informan o no prestan servicios digitales de biblioteca como un valor más, ya sea a través de Internet o de intranet, a sus usuarios reales y potenciales.

Scire. $13: 2$ (jul.-dic. 2007) 59-65. ISSN 1135-3716. 
En relación con los servicios ofrecidos, ya hemos podido comprobar que los más frecuentes son los de acceso, seguidos de los de información general sobre la biblioteca hospitalaria, los relacionados con el préstamo y, en menor medida, de otros servicios. No es menos cierto que los servicios que presta cada biblioteca dependen de la finalidad que tenga y los medios disponibles.

Por este motivo, unido al de prestar mejores servicios, queremos animar al personal bibliotecario de dichas instituciones a fomentar su visibilidad a través de los servicios innovadores ofrecidos a través de Internet, de una forma normalizada en cuanto a la construcción de la página (siguiendo unos criterios de usabilidad y accesibilidad), utilizando además algún modelo de servicios digitales como podrían ser determinadas bibliotecas digitales o virtuales: la del hospital universitario 12 de Octubre, la del complejo hospitalario Carlos Haya, etcétera.

Se ha observado durante el análisis cómo aquellas instituciones vinculadas con la docencia y la investigación que cuentan con una trayectoria amplia en este sentido suelen tener unas mejores páginas webs, no solo para el hospital, sino también para los servicios de apoyo, como se denominan en algunos hospitales los servicios de biblioteca o centro de documentación clínica.

\section{Referencias}

Arévalo Molina, J., et ál. (2005). Servicios bibliotecarios en las bibliotecas digitales. http://www.biblioteca.uclm.es/Articulos/malaga2.pdf (2005-05-28).

Barry, C. (1999). Las habilidades de información en un mundo electrónico: la formación investigadora de los estudiantes de doctorado. // Anales de Documentación. 2 (1999). http://www.um.es/fccd/anales/ad02/AD12-1999.PDF (2004-12-22).

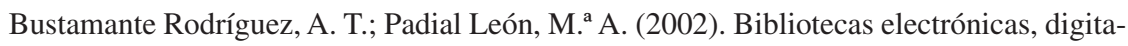
les y virtuales: conceptualización del nuevo modelo de biblioteca. // XII Jornadas Bibliotecarias de Andalucía (Jerez de la Frontera, 28-30 de mayo de 1998). Málaga: Asociación Andaluza de Bibliotecarios, 2002. 279-288.

Brophy, P.; Coulling, K. (1996). Quality management for information and library managers. Aldershot: Gower, 1996.

Crosby, P. (1993). Hablemos de calidad. Madrid: MacGraw-Hill, 1993.

García Marco, F. J. (2002). La biblioteca digital. // López Yepes, José (coord.). Manual de ciencias de la documentación. Madrid: Pirámide, 2002.

Gutiérrez, U.; Blanco, A.; Vivas, M.; Calvo, A.; Cámara, J. M. de la (2003). Ser o no ser y estar o no estar en Internet. // X Jornadas Nacionales de Información y Documentación en Ciencias de la Salud (Málaga, 13-15 de noviembre de 2003). http://www.jornadas bibliosalud.net/comunicaciones/cc28.rtf (2005-05-28). Disponible también en http://eprints.rclis.org/11607/.

Jovell, Albert J. (2005). Bibliotecas en ciencias de la salud: el futuro de la gestión digital del conocimiento. http://www.imim.es/quark/num14/014024.htm (2005-05-28).

Scire. 13 : 2 (jul.-dic. 2007) 59-65. ISSN 1135-3716. 
Lancaster, F. W. (2001). Procesamiento de la información científica. Madrid: Arco/Libros, 2001.

Ministerio de Sanidad y Consumo. Catálogo Nacional de Hospitales: información por comunidad autónoma. http://www.msc.es/Diseno/sns/sns sistemas informacion.htm (2005-05-28). Posteriormente el URL cambió a http://www.msc.es/ciudadanos/ prestaciones/centrosServiciosSNS/hospitales/home.htm.

Pinto Molina, M. (1994). Indicadores de calidad descriptiva en la gestión de los procesos analítico-documentales. // FESABID, 1994.

Pinto Molina, M. (1998). Gestión de la calidad en documentación. // Anales de documentación. 1 (1998) 171-183.

Lancaster, W.; Pinto Molina, M. (coords.) (2001). Procesamiento de la información científica. Madrid: Arco/Libros, 2001.

Ishikawa, K. ¿Qué es el control de calidad?: la modalidad japonesa. Barcelona: Norma, 1990. 